\title{
Computational and transcriptional evidence for microRNAs in the
} honey bee genome

\author{
Daniel B Weaver ${ }^{\not *}$, Juan M Anzola ${ }^{\not \dagger}$, Jay D Evans ${ }^{\not \neq}$, Jeffrey G Reid ${ }^{\ltimes}$, \\ Justin T Reese ${ }^{\dagger}$, Kevin L Childs ${ }^{\dagger * *}$, Evgeny M Zdobnov ${ }^{\uparrow+\dagger}$, \\ Manoj P Samanta ${ }^{\ddagger}$, Jonathan Miller" and Christine G Elsik ${ }^{\dagger}$
}

\begin{abstract}
Addresses: *Bee Power, LP, Lynn Grove Road, 16481 CR 319, Navasota, TX 77868 USA. ${ }^{\dagger}$ Department of Animal Science, Texas A\&M University, College Station, Texas 77843, USA. ${ }^{*}$ Bee Research Laboratory, USDA-ARS, BARC-E, Beltsville, MD, USA. §WM Keck Center for Interdisciplinary BioScience Training, Houston, TX 77005, USA. "European Molecular Biology Laboratory, Meyerhofstr., Heidelberg, Germany. ${ }^{¥}$ Systemix Institute, Los Altos, CA 94024, USA. " Department of Biochemistry, Baylor College of Medicine, Houston, TX 77030, USA. ${ }^{* *}$ The Institute for Genome Research, Rockville, MD 20850, USA. ${ }^{+\dagger}$ Department of Genetic Medicine and Development, University of Geneva Medical School (CMU), rue Michel-Servet 1, 1211 Geneva 4, Switzerland.
\end{abstract}

$\star$ These authors contributed equally to this work.

Correspondence: Christine G Elsik. Email: c-elsik@tamu.edu

Published: I June 2007

Genome Biology 2007, 8:R97 (doi:10.1 186/gb-2007-8-6-r97)

The electronic version of this article is the complete one and can be found online at http://genomebiology.com/2007/8/6/R97
Received: II August 2006

Revised: 13 December 2006

Accepted: I June 2007

(c) 2007 Weaver et al.; licensee BioMed Central Ltd.

This is an open access article distributed under the terms of the Creative Commons Attribution License (http://creativecommons.org/licenses/by/2.0), which permits unrestricted use, distribution, and reproduction in any medium, provided the original work is properly cited.

\begin{abstract}
Background: Non-coding microRNAs (miRNAs) are key regulators of gene expression in eukaryotes. Insect miRNAs help regulate the levels of proteins involved with development, metabolism, and other life history traits. The recently sequenced honey bee genome provides an opportunity to detect novel miRNAs in both this species and others, and to begin to infer the roles of miRNAs in honey bee development.
\end{abstract}

Results: Three independent computational surveys of the assembled honey bee genome identified a total of 65 non-redundant candidate miRNAs, several of which appear to have previously unrecognized orthologs in the Drosophila genome. A subset of these candidate miRNAs were screened for expression by quantitative RT-PCR and/or genome tiling arrays and most predicted miRNAs were confirmed as being expressed in at least one honey bee tissue. Interestingly, the transcript abundance for several known and novel miRNAs displayed caste or age-related differences in honey bees. Genes in proximity to miRNAs in the bee genome are disproportionately associated with the Gene Ontology terms 'physiological process', 'nucleus' and 'response to stress'.

Conclusion: Computational approaches successfully identified miRNAs in the honey bee and indicated previously unrecognized miRNAs in the well-studied Drosophila melanogaster genome despite the $\mathbf{2 8 0}$ million year distance between these insects. Differentially transcribed miRNAs are likely to be involved in regulating honey bee development, and arguably in the extreme developmental switch between sterile worker bees and highly fertile queens. 


\section{Background}

MicroRNAs (miRNAs) play pivotal roles in diverse biological processes through post-transcriptional regulation of gene expression. These short (approximately 22 nucleotide (nt)) non-coding RNAs repress protein synthesis by binding to partially complementary sites in the 3 ' untranslated regions (UTRs) of target genes [1-3]. MiRNAs affect biological phenomena such as cell proliferation, embryo and tissue differentiation [4], morphological change [5], and apoptosis, aging and life span [6]. Overall, miRNAs appear to regulate much of the coding transcriptome, influencing the spatial and temporal expression patterns of thousands of genes in plants, nematodes, insects, and vertebrates $[7,8]$. The pervasive influence of miRNAs exerts strong selective pressures on nucleotide sequences. Either positive selection for, or negative selection against, miRNA target sites can be detected in the 3' UTRs of most genes $[9,10]$.

MiRNA sequences are often, but not invariably, highly conserved across great evolutionary distances, allowing identification of nearly identical short oligonucleotides that affect gene expression in species as divergent as worms and man [11]. This extraordinary sequence conservation may be indicative of extraordinary functional conservation, or some other exceptional evolutionary constraint. For instance, because a single miRNA may regulate hundreds of genes, mutation of a mature miRNA sequence could pleiotropically affect the expression breadth and specificity of many gene targets [12]. Thus, preservation of miRNA function in the wake of miRNA mutation would require coordinated compensatory mutation of each of its target's 3' UTRs - predicted to be an exceedingly rare confluence of events. Consequently, the sequence, structure and some functions of miRNAs may be conserved [13], while the specific gene targets and regulatory networks of particular miRNAs may exhibit significant interspecies variation [14].

The recently sequenced honey bee genome [15] provides an opportunity to detect novel miRNAs in this species and others, and to begin to infer the roles of miRNAs in key life history traits of honey bees, such as the development of fertile as well as sterile ('worker') individuals. Here we present the results of three independent computational surveys and transcriptional evidence for known and novel miRNAs. We suggest several novel miRNA candidates in honey bees. Some of these novel miRNAs appear to have been overlooked in analyses of the well-studied insect Drosophila melanogaster and other genomes.

\section{Results}

\section{Computational identification of putative miRNAs}

We exploited the whole genome assembly of the honey bee to predict candidate miRNAs. Three non-exclusive sets of miRNA candidates were compiled. First, honey bee sequences homologous to miRNAs listed in miRBase [16] were identified (HOM). Second, microconserved-sequence elements (MCEs), continuous sequences of lengths 22 through $29 \mathrm{nt}$ that are common to and precisely conserved in all three of the Apis mellifera, D. melanogaster and Anopheles gambiae genomes, were catalogued [17].

Finally, slightly longer bee sequences (75-90 nt) sharing structural features characteristic of miRNAs and aligning well with similar sequences in Drosophila - an approach we call stem-loop scanning (SLS) - identified another set of putative honey bee miRNAs. This approach does not simply flag regions with propensity to form stem loop structures of appropriate length because there are thousands of such regions in the $235 \mathrm{Mb}$ of the sequenced honey bee genome. Instead, Smith-Waterman alignments to regions of the Drosophila genome likely to form pre-miRNA structures were used to filter and refine the list of putative SLS candidates in honey bee.

Each putative miRNA precursor (pre-miRNA) identified by any method was folded to verify the thermodynamic propensity of the pre-miRNA sequence to adopt appropriate hairpin secondary structure - and to verify that the mature miRNA resided in the stem of the hairpin. We identified putative canonical honey bee miRNAs, but the MCE and SLS methods also suggested a number of possible new miRNAs, present but previously unrecognized in other genomes.

Consolidation of output from the MCE and homology-based miRNA search methods provided a final set of 65 unique miRNA candidate loci with 66 unique predicted miRNA models for experimental evaluation - including the best 25 predictions generated by MCE. This final set of 65 miRNA loci included 6 putative miRNAs identified by either homology or MCE methods, but also by the SLS process. However, none of the candidates identified only by SLS were among the final set of 65 , or tested for expression in this study. Honey bee miRNA candidates, including some potentially novel miRNAs and a few honey bee orthologs of known miRNAs, are listed in Additional data file 1 . There were two variant mature and precursor miRNA models predicted by MCE and HOM for one of the predicted miRNA loci. For each candidate honey bee miRNA model, Additional data file 1 gives the prediction method (HOM, MCE and/or SLS), miRBase designation if available, sequences of the putative mature honey bee miRNA and putative precursor region, genomic coordinates of each occurrence of mature and putative precursor miRNA sequences within the bee genome assembly release 4, location relative to coding sequence (CDS) of the honey bee official gene set [18] (intergenic, intronic, or overlapping a CDS), GC content of the GC content domain in which the miRNA is embedded (described in [15]), and folding energies. Folded precursors for some of the novel miRNAs are shown in Additional data file 8 . 
Validation of honey bee miRNA candidates by RT-PCR A variety of techniques are available for miRNA detection and validation, including hybridization techniques such as Northern blots and techniques using PCR (reviewed in [19]). We employed the RT-PCR technique described by Shi and Chang [20] to verify transcription of many of the candidate honey bee miRNAs we describe. In brief, this protocol invokes the polyadenylation of extracted RNA (in our case, after sizeselection for small RNA species by either glass-fiber substrate binding or separation using polyacrylamide gel electrophoresis) followed by reverse-transcription primed by a poly(T) adapter. MiRNA-specific forward primers are then paired with a primer complementary to the RT adaptor for quantitative PCR amplification.

Table 1 shows normalized expression levels across a pool of larvae and adult bee samples for 30 candidate miRNAs. Some candidates were queried with multiple primers in order to test for strand-based expression and to distinguish between expression of precursor and mature miRNA sequences, leading to a total of 45 presented primers. Another 23 primers either generated artifactual PCR products in water or oneprimer controls, or failed tests of amplification linearity. In general, candidates tested with forward and reverse primers showed much higher expression of one strand. As a methodological control showing strand specificity, primers for two variants of U4 spliceosome RNA (C5581a and $\mathrm{C}_{5581 \mathrm{~b}}$ ) showed strong expression in the predicted reverse direction while a forward-oriented primer for $\mathrm{C} 5581 \mathrm{~b}$ showed almost no expression. Expression for this locus was marginal when the narrow (enriched for 18-30 nucleotide (nt) species) RNA pool was queried. Primers that matched mature miRNAs tended to generate stronger signal, especially when testing the gel-purified (18-30 nt) RNA extractions. Alignments of the tested primers to candidate miRNAs appear in Additional data file 2 and a gel showing quantitative RT-PCR (qRT-PCR) products from the $18-30$ nt size selected RNA is found in Additional data file 9.

We found 25 potentially novel miRNAs by MCE, of which 17 were tested by qRT-PCR. Twelve of these were expressed in one or more tissues, stages, castes or pooled RNA samples, while four had no detectable expression (C2327, C4131, C5267 and C6617). Nevertheless, three of the RT-PCR negative candidates showed evidence of transcription in the tiling array data (C4131, C6617 and C5267).

$\mathrm{C}_{5152 \mathrm{a}}$ and $\mathrm{C}_{5152 \mathrm{~b}}$ are discrete miRNA predictions in physical proximity on opposite strands (shown in Additional data file 1) and both yield good hairpin predictions (Additional data file 8). $\mathrm{C}_{5152 \mathrm{~b}}$ is similar, but not identical, to Drosophila dre-ame-190. :Expression of $\mathrm{C}_{5152 \mathrm{a}}$ and $\mathrm{C} 5152 \mathrm{~b}$ by RT-PCR was tested using multiple primers, and both F- and D+ primers showed expression (Additional data file 3 ). Primers F- and $\mathrm{D}+$ were designed to amplify the mature miRNAs predicted for $\mathrm{C}_{5152 \mathrm{a}}$ and $\mathrm{C}_{5152 \mathrm{~b}}$, respectively (Additional data file 2).
However, the complex overlap and antisense orientation of these two predictions, and binding sites for both F- and D+ within each of $\mathrm{C}_{5152 \mathrm{a}}$ and $\mathrm{C}_{5152 \mathrm{~b}}$, prevent us from excluding the possibility that only one is actually expressed in both sense and antisense orientations.

Overall, we provide evidence of transcription for most of the novel MCE predictions, including roughly two-thirds of novel candidates amenable to RT-PCR testing. Predicted expression levels were correlated between assays involving RNA extracts biased toward small species using either selective precipitation or electrophoretic separation (Table 1; Additional data file 3). Additional candidates will likely be confirmed as having transcription using other techniques and honey bee tissues or life stages.

\section{Validation of miRNA candidates by whole genome tiling array}

We also analyzed the results of two whole-genome honey bee tiling array experiments for evidence that our candidate miRNAs were expressed. Using RNA pooled from multiple tissues and stages, genome-wide transcription, including intergenic regions, was evaluated by hybridization to 36-mer probes. Two strand-specific 36 nt oligonucleotide probes for every 46 $\mathrm{bp}$ of the honey bee genome were arrayed. The whole genome tiling array was hybridized in two separate experiments with two different pooled polyadenylated RNA samples; but the second experiment contained pooled RNA enriched for brain and thorax.

For each candidate miRNA, tiling probes in a genomic region containing its precursor sequence flanked by 50 bases on both 5 ' and 3' ends were examined. A miRNA was considered expressed if at least one probe within the chosen region measured signal above $90 \%$ of all tiling probes from the entire genome. Twenty-six miRNAs, listed in Additional data file 6, measured strong signal in either of the tiling array experiments and six in both. Among the latter six, C4222, C6617 and ame-mir-100 exhibited differential signal strength in the two tiling array experiments.

Tiling array experiments measure genome-wide expression patterns in an unbiased manner. In several organisms, signals from tiling arrays were observed in numerous noncoding regions of the genome, suggesting the presence of noncoding RNA, including tRNAs. Notably, tRNAs are approximately the same size as miRNA precursors [21]. However, neither pre-miRNAs nor mature miRNAs will be polyadenlylated. Thus, use of polyA RNA in these experiments therefore biased the RNA samples against mature miRNAs. Consequently, failure of some RT-PCR validated miRNAs to be detected as tiling array signals is not surprising. Conversely, there was difficulty in assigning statistical significance to the observed tiling array signals because the array experiments were designed to detect longer protein-coding genes. Therefore, there were too few probes (approximately 3-4) for each 
Table I

\begin{tabular}{|c|c|c|c|c|c|c|}
\hline Locus & miRBase ID & Primer ID & Orientation & Location & Expression (not size selected) & Expression (size selected) \\
\hline ame-mir-I & ame-mir-I & ame-mir-I.F & $\mathrm{F}$ & M & 0.06 & N/A \\
\hline ame-mir-I & ame-mir-I & amirl.F & $\mathrm{F}$ & $P$ & 1.05 & 1.28 \\
\hline ame-mir-124 & ame-mir-124 & miR-I24M35I & $\mathrm{F}$ & M & 2.09 & 0.69 \\
\hline ame-mir-124 & ame-mir-124 & miR-I24M35IR & $R$ & M & 0.40 & 0.07 \\
\hline ame-mir-2-I & ame-mir-2-I & ame-mir-2+.F & $\mathrm{F}$ & M & 7.81 & 13.55 \\
\hline ame-mir-2-2 & ame-mir-2-2 & & & & & \\
\hline ame-mir-2-3 & ame-mir-2-3 & & & & & \\
\hline ame-mir-2-I & ame-mir-2-I & mir-2:I.I:10|7|2.F & $\mathrm{F}$ & $P$ & 0.06 & 0.02 \\
\hline ame-mir-278 & ame-mir-278 & ame-mir-278.F & $\mathrm{F}$ & M & 0.37 & 0 \\
\hline ame-mir-7 & ame-mir-7 & ame-mir-7.F & $\mathrm{F}$ & $M$ & 17.95 & 3.16 \\
\hline ame-mir-7 & ame-mir-7 & miR-7MIII2R & $\mathrm{F}$ & M & 0.06 & N/A \\
\hline ame-mir-9a & ame-mir-9a & ame-mir-9a.F & $\mathrm{F}$ & M & 23.69 & 9.58 \\
\hline ame-mir-9b & ame-mir-9b & ame-mir-9b & $\mathrm{F}$ & M & 0.64 & 1.95 \\
\hline ame-mir-iab-4 & ame-mir-iab-4 & ame-miriab4.F & $\mathrm{F}$ & $P$ & 0.24 & 0.06 \\
\hline ame-mir-10 & ame-mir-10 & ame-miR-10 & $\mathrm{F}$ & $M$ & 0.09 & N/A \\
\hline ame-mir-279 & ame-mir-279 & miR-279M34I & $\mathrm{F}$ & $M$ & 5.53 & 1.04 \\
\hline ame-mir-279 & ame-mir-279 & miR-279M34IR & $\mathrm{R}$ & M & 0.56 & N/A \\
\hline ame-mir-283 & ame-mir-283 & HCmir-283.F & $\mathrm{F}$ & $M$ & 23.69 & 17.88 \\
\hline ame-mir-7I & ame-mir-7I & miR-7I.R & $\mathrm{R}$ & $M$ & 1.70 & 1.38 \\
\hline ame-mir-87-2 & ame-mir-87-2 & mir-87:13.I2:403730.F & $\mathrm{F}$ & $P$ & 0.43 & 0.91 \\
\hline ame-bantam & ame-bantam & banM365 & $\mathrm{F}$ & $M$ & 0.16 & 0.06 \\
\hline $\mathrm{Cl} 504$ & ame-mir-925 & $\mathrm{Cl} 504 . \mathrm{F}$ & $\mathrm{F}$ & $M$ & 0.11 & 0.30 \\
\hline C2989 & ame-mir-926 & C2989.F & $\mathrm{F}$ & M & 31.25 & N/A \\
\hline C3345 & ame-mir-927 & contig3345.R & $\mathrm{R}$ & M & 0.17 & 3.63 \\
\hline C4222 & ame-mir-928 & C4222.F & $\mathrm{F}$ & M & $N / A$ & 3.39 \\
\hline C5152a & ame-mir-190* & C5I52a.F & $\mathrm{F}$ & O3 & 0.52 & 0.64 \\
\hline C5152a & ame-mir-190* & $D+$ & $\mathrm{R}$ & 0 & 1.29 & 0.20 \\
\hline $\mathrm{C} 5152 \mathrm{~b}$ & ame-mir-190 & C5I52b.F & $\mathrm{F}$ & $P$ & 0 & 0 \\
\hline C5I52b & ame-mir-190 & F- & $\mathrm{R}$ & P & 76.96 & 25.28 \\
\hline C5303 & ame-mir-137 & C5303.F & $\mathrm{F}$ & O3 & 2.96 & 0.74 \\
\hline C5303 & ame-mir-137 & $\mathrm{C}^{+}$ & $\mathrm{R}$ & O3 & 0.15 & 0.02 \\
\hline C5560 & ame-mir-929 & C5560.F & $\mathrm{F}$ & O & 955,568 & 45,073 \\
\hline C5560 & ame-mir-929 & A- & $\mathrm{R}$ & $\mathrm{O} 3$ & 0.01 & 0 \\
\hline C5560 & ame-mir-929 & C5560.R & $\mathrm{R}$ & M & 0.00 & 0 \\
\hline C5599 & ame-mir-930 & C5599b.F & $\mathrm{F}$ & $M$ & 0.54 & 0.08 \\
\hline C689 & ame-mir-932 & amirl.R & $\mathrm{F}$ & $M$ & 0.23 & 0.28 \\
\hline C689 & ame-mir-932 & contig689.F & $\mathrm{F}$ & $P$ & 20.62 & $N / A$ \\
\hline C689 & ame-mir-932 & C689.F & $\mathrm{R}$ & $M$ & 0.16 & $N / A$ \\
\hline C689 & ame-mir-932 & $\mathrm{E}^{+}$ & $R$ & $M$ & $N / A$ & 1.69 \\
\hline$C 2187 \dagger$ & & $\mathrm{C} 2187 . \mathrm{F}$ & $\mathrm{F}$ & $M$ & 0.98 & $N / A$ \\
\hline $\mathrm{C} 2370^{\dagger}$ & & $\mathrm{C} 2370 . \mathrm{F}$ & $\mathrm{F}$ & $M$ & 8.37 & $N / A$ \\
\hline C558I $\mathrm{a}^{\ddagger}$ & & C558Ia.R & $\mathrm{R}$ & M & 20.62 & N/A \\
\hline C558Ib $\neq$ & & C558Ib.F & $\mathrm{F}$ & $M$ & 0.07 & 0 \\
\hline C558Ib $\ddagger$ & & C558Ib.R & $\mathrm{R}$ & M & 22.10 & N/A \\
\hline
\end{tabular}

Orientation is on predicted miRNA ( $F$, forward; $R$, reverse). Location is within: mature miRNA (M); precursor sequences (P); overlapping mature miRNA with 3' primer end within mature sequence $(\mathrm{O})$; overlapping mature miRNA but with $3^{\prime}$ primer end in precursor $(\mathrm{O} 3)$. Expression levels for pooled queen and worker samples are described in the text. The last two columns are normalized expression estimates for pooled RNA that either had or had not been size-selected by PAGE to include sizes from I8-30 nt. *C5I52a is the reverse complement of ame-mir- 190 . $+C 2187$ and C 2370 met thermodynamic criteria, but did not meet miRBase folding criteria. $\neq$ Denotes $U 4$ spliceosome RNA. The expression levels are scaled to the average of all primers.

miRNA precursor, and typically only one of these probes showed strong signals. The significance of tiling array results is higher for the six miRNAs displaying strong signals in both experiments $\mathrm{A}$ and $\mathrm{B}$, and for the twelve miRNA candidates that also exhibited RT-PCR results consistent with transcrip- tion. However, differential signal for three of the tiling array positive miRNA candidates suggests that those miRNAs (C6617, C4222 and ame-mir-100) may have roles in bee brain or thorax. 


\section{Caste-, tissue- and age-related miRNA expression correlations}

We hypothesized that miRNAs might be involved in the dramatic developmental fate changes associated with the switch from a reproductive female to a sterile worker female caste. Accordingly, RNA was isolated from various tissues and stages of both queen and worker honey bees and characterized by RT-PCR. Figure 1 and Additional data file 10 contrast expression levels for a subset of the candidate miRNA loci in adult head, thorax, abdomen and whole pupae, for both queens and workers. Several candidates showed differential expression between queens and workers in the abdomen, arguably the body part that is physiologically most distinct between these castes due to their different fecundity. Candidate loci ame-mir-9a, C3345, and C5152 were more strongly expressed in worker abdomens, while $\mathrm{C}_{150} 4$ and ame-mir-71 were more strongly expressed in queen abdomens. Ame-mir71 also had far stronger expression in developing (pupal) workers than in queens and in worker thoraces. A more complete summary of RT-PCR experiments for this subset is shown in Additional data file 4. In agreement with our hypothesis that computationally predicted honey bee miRNAs could be implicated in bee development, and particularly in the changes that characterize alternative fates of worker and queen, many miRNAs display tissue, stage or casterelated expression patterns. Additional data files provide the values of RT-PCR transcription estimates for pooled RNA (Additional data file 3) and additional queen/worker samples (Additional data file 4), primer sequences employed for experimental evaluation (Additional data file 5), and alignments of the primers to the precursor sequences (Additional data file 2).

\section{Intronic miRNAs and host genes}

MiRNAs are often clustered within the genomes of mammals and flies, and this clustering is often associated with co-transcription of miRNAs and genes with which they are in close proximity [22]. The co-transcription of miRNAs and nearby genes may also reflect coordinate regulation of miRNAs and nearby genes. In particular, intronic miRNAs are often, though not invariably, coordinately expressed with their host gene and transcribed as a single primary transcript [23]. In support of the postulated role of miRNAs in regulating the alternative developmental trajectories associated with caste differentiation, we examined the functional role of honey bee official gene set genes in which intronic honey bee miRNAs are embedded [18]. Given the paucity of direct functional evidence for most genes in honey bees, we relied upon a comprehensive set of computational orthologs described elsewhere [15]. We discovered several notable relationships that will merit additional investigation. First, there were associations with fundamental cellular machinery of growth and development. Ame-mir-34, ame-mir-277 and ame-mir-317 all occupy intron 3 of GB10191. GB10191 is the ortholog of Rbp8 in Drosophila, and RPB8 in humans - part of the RNA polymerase II core complex and intimately involved in all transcriptional activity. Similarly, ame-mir-279 is embedded within intron 3 of GB12486, the honey bee DNA polymerase- $\alpha$ primase.

Intriguingly, the functional processes of other genes hosting intronic miRNAs suggest some bee miRNAs may be implicated in important but more complex caste differences. For instance, novel candidate miRNA C689 is found within GB10066, the bee ortholog of neuroligin, implicated in nervous system development. Novel miRNA C1504 is embedded in GB11212, whose Drosophila ortholog is involved in the dorsal/ventral patterning, expressed in wing discs, and negatively regulated by Ultrabithorax. Candidate $\mathrm{C}_{5267}$ is contained in GB15446, whose Drosophila homologs are regulators of transcription from RNA polymerase II promoters, and involved in eye development and other morphogenic interactions. Novel candidate $\mathrm{C}_{5599}$ is found within GB14516, the ortholog of Dll (Distalless), which has transcription factor activity and is intimately involved in proximal/distal pattern formation and morphogenesis, especially antennae and genitalia formation. Bee miRNAs may also be involved in programming behavioral response repertoires, as GB15597 harbors miRNA C4222, and its fly ortholog is eag, implicated in behavioral responses, including sensory perception of smell and flight.

\section{Gene Ontology analysis}

We reasoned that an analysis of overrepresented Gene Ontology (GO) [24] terms associated with genes near miRNAs might offer additional insights into function for some bee miRNAs, and allow us to examine broad patterns of functional conservation between bee miRNAs and Drosophila miRNAs. We first determined the GO slim terms (a more general subset of GO terms) associated with the Drosophila ortholog of each bee gene [15]. Then using GeneMerge [25], we determined GO slim terms that were overrepresented among the set of bee genes occurring $<10,<20,<50$ or $<100$ $\mathrm{kb}$ from a predicted mRNA, compared with the set of all bee genes with Drosophila orthologs. Because some bee genes have multiple orthologs to Drosophila, and to ensure that our GO enrichment analysis was not biased by random selection of one to many fly orthologs of bee gene near miRNAs, we performed ten GeneMerge replicate experiments at each distance and report only GO terms whose Bonferroni corrected E-socres were less than 0.05 in all ten replicates.

GO analysis revealed the following: 'Physiological process' as the only GO term overrepresented among genes $<10 \mathrm{~kb}$ from bee miRNAs in every replicate experiment; 'Response to stess' overrepresented in every replicate experiment for genes $<20 \mathrm{~kb}$ from bee miRNAs; no GO term overrepresented in every replicate $<50 \mathrm{~kb}$ from bee miRNAs; 'Nucleus' overrepresented in every replicate $<100 \mathrm{~kb}$ from bee miRNAs. Running GeneMerge on a negative control set consisting of randomly selected bee genes yielded no GO terms with significant Bonferroni corrected E-scores. 


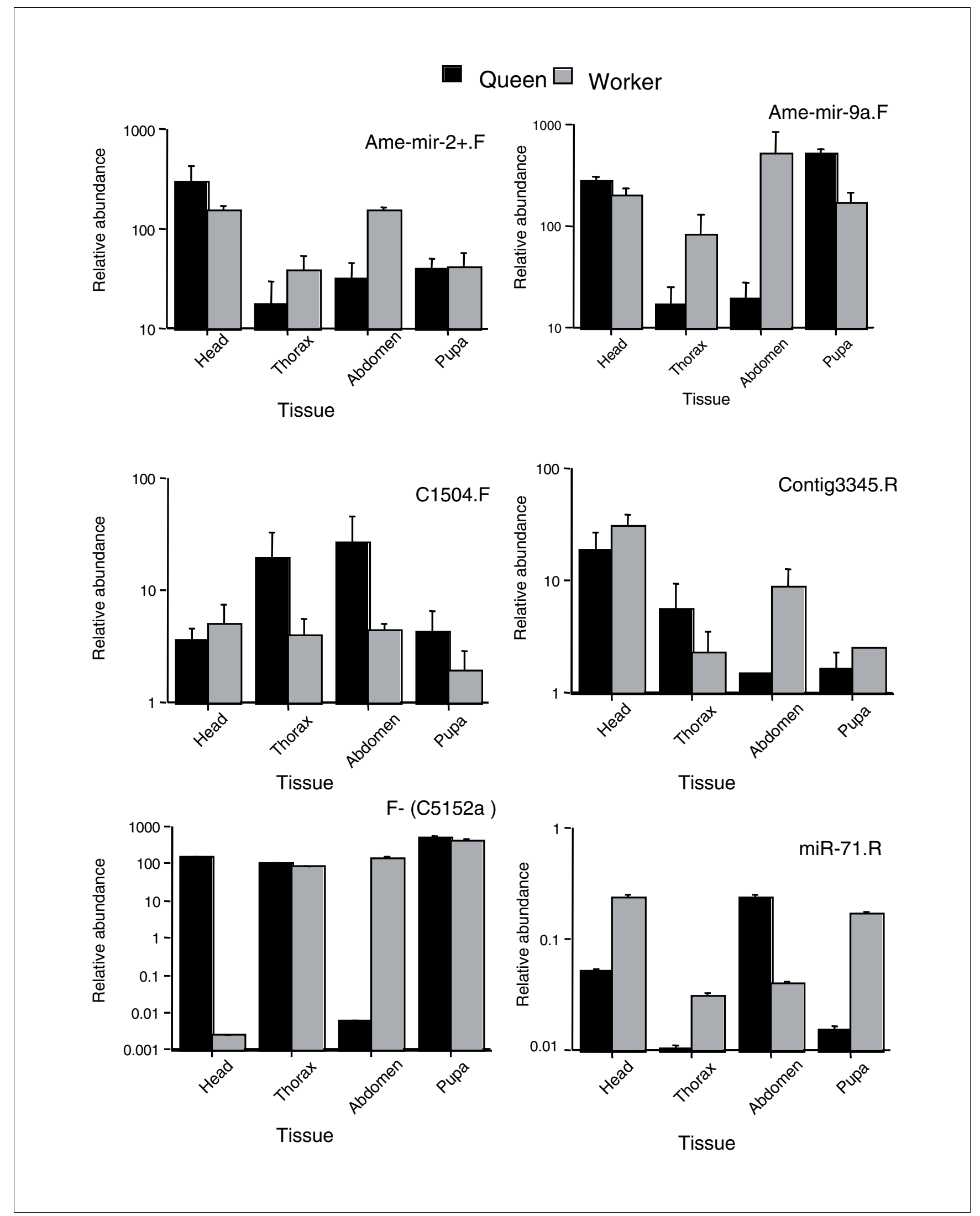

Figure I (see legend on next page) 
Figure I (see previous page)

Normalized expression across worker and queen samples for six miRNA candidates. Values indicate relative expressions levels as log ${ }_{10}$ Scale, with SD for three sample replicates, as described in the text. Primer IDs are indicated.

To compare GO terms associated with these miRNAs in bee and fly, we conducted a similar analysis of Drosophila genes near miRNAs. We obtained GO slim terms associated with Drosophila genes occurring $<10,<20,<50$ or $<100 \mathrm{~kb}$ from Drosophila orthologs of these bee miRNAs, and ran GeneMerge to find overrepresented GO terms. As before, only GO terms whose Bonferroni corrected E-scores were less than 0.05 in all ten replicate experiments are reported. The GO experiment data are summarized in Additional data file 7. Interestingly, the GO term 'Physiological process', which was overrepresented among bee genes $<10 \mathrm{~kb}$ from miRNAs was also overrepresented among Drosophila genes $<20,<50$ and $<100 \mathrm{~kb}$ from miRNAs. As before, running GeneMerge on a negative control set consisting of randomly selected Drosophila genes yielded no GO terms with significant Bonferroni corrected E-scores.

Compared to bee, there were far more GO terms that were significantly enriched among genes near miRNAs in the Drosophila genome. For example, four GO slim terms ('Development', 'Morphogenesis', 'RNA binding' and 'Signal transduction') were overrepresented in all replicates at every distance in Drosophila, and there were 29 GO terms significantly enriched among genes $<100 \mathrm{~kb}$ from fly miRNAs (Additional data file 7). In contrast, in the bee genome, there were no GO terms enriched at every distance, and only 1 GO term ('Nucleus') enriched among genes $<100 \mathrm{~kb}$ from bee miRNAs. This disparity between bee and Drosophila is likely caused by the increased sensitivity in the Drosophila experiment compared to the bee experiment. The Drosophila experiment used Drosophila GO annotations directly, whereas the bee experiment relied on the existence and detection of Drosophila orthologs for each bee gene.

\section{Discussion}

The honey bee genome [15] offers a rich resource for investigation of the genomic networks and emergent systems that characterize sociality and enable coherent operation of the complex web of interactions in the hive. However, the significant level of sequence divergence of honey bee from Drosophila and mosquito, and the absence of closely related genome sequences suitable for phylogenetic shadowing can impede genomic comparisons involving bees. We turned evolutionary distance to our advantage, reasoning that strongly conserved sequences in an appropriate length range (MCEs) might represent previously undiscovered miRNAs (the MCE algorithm) [17]. In addition, we exploited the secondary structure characteristics of most confirmed miRNAs, and the conservation of core microprocessor components in bee, like Drosha, to identify other candidates that would adopt pre-
miRNA hairpin structures, and produce significant SmithWatermann alignments between putative bee and Drosophila miRNAs (the SLS algorithm).

Among those novel miRNA predictions we tested, we observed only one false positive candidate identification by MCE. C5581 was predicted as a miRNA, but that sequence is homologous to a U4 splicing RNA. There was one case in which two methods predicted slightly different miRNAs at overlapping genomic coordinates. Mature ame-mir-137, identified by HOM, is completely identical over the $22 \mathrm{nt}$ that it overlaps with the $27 \mathrm{nt}$ of mature $\mathrm{C} 5303$, predicted by MCE. We observed two cases where different miRNA predictions occurred at overlapping genomic coordinates, but the opposite strand: C5152a/C5152b (primers F- and D+) and amemir-9b/ame-mir-79. In both cases, at least one of the opposing strand pair was identical or similar to a known mature miRNA. Predicted ame-miR-9b and ame-mir-79 are identical to known miRNAs. Predicted mature $\mathrm{C}_{5152} \mathrm{~b}$ is similar, but not identical to Drosophila dme-mir-190; $\mathrm{C}_{5152 b}$ is longer than dme-mir-190, and differs at only three nucleotides internally. These may be examples of miRNA sense/antisense transcription.

The SLS output contained five predictions with significant similarity to the HOM output (ame-mir-13a, ame-mir-276, ame-mir-305, ame-mir-92 and ame-mir-9a) and only two predictions with significant similarity to the top $25 \mathrm{MCE}$ candidates, both of which were variants of C5152. Of these SLS predictions, only ame-mir-9a and $\mathrm{C}_{5152}$ were tested for expression by RT-PCR, and both were validated. The tiling array evidence we accumulated also suggests that mir-305 is expressed. The SLS output included several novel pre-miRNA predictions that contained apparent repeat motifs and are unlikely to be true miRNAs. However, other SLS candidates may represent new miRNAs and future experiments will more systematically assess evidence of expression for some of them.

We detected transcription of mature miRNAs as well as some pre-miRNAs. Generally, putative mature miRNA transcript abundance exceeded the level of precursor transcripts. Primers for mature miRNAs also tended to show the strongest effects of transcript direction (for example, ame-mir-279; Table 1), and retained strand-specific expression levels when the 18-30 nt RNA pool was assayed. Nevertheless, tests at a number of candidate miRNAs indicated fairly similar $\left(<5^{-}\right.$ fold difference) transcription levels for both RNA strands (for example, ame-mir-1). Due to the small sample sizes, we have highlighted only the more extreme expression differences, although, as has been shown in expression studies of protein- 
encoding transcripts in bees, even subtle differences in transcript abundance could play important roles in development. It is possible that actual mature miRNA for those candidates that did demonstrate expression may differ slightly from the mature miRNA we predicted. For example, a variant of the primer for candidate ame-mir-7 (ame-mir-7.F) indicated a very strong transcript level, while a primer with one more 3' nucleotide (T; miR-7M112R) gave no product. Thus, we showed that our RT-PCR technique was very sensitive to small primer sequence differences, as shown in plant miRNAs by Shi and Chang [20].

Likewise, the strongest expression product observed (C5560F) was primed by a forward primer that stopped one base short of the 5 ' end of the predicted mature miRNA (Additional data file 2). Because it is possible that the actual novel mature miRNA sequences may differ slightly from the sequence of the candidate mature miRNA primers we tested, we cannot unequivocally reject those candidate miRNAs for which we did not obtain reproducible expression patterns.

Honey bee genomic study is still young, but initial observations offer some clarity and focus for further investigation. First, with a few notable exceptions (for example, odorant receptor genes and genes involved with innate immunity), there are as yet few potential relationships between gross genomic features and the social organization of bees [15]. In fact, the emergence of social life and its manifestation in bees may rely mainly on fairly subtle genomic interactions that affect gene network organization, regulation and expression patterns. In support of this hypothesis, previous work suggests that the development of distinct reproductive castes (workers and queens) in honey bees reflects the differential regulation of well-established developmental genes, rather than that of a parallel set of caste-specific genes [26,27].

We submit that miRNAs and their combinatorial interactions with overlapping and independent target gene sets may offer a tractable means to aid the evolution of sociality, by stabilizing the alternative developmental programs that generate distinct castes from a uniform genetic groundplan. Thus, the evolution of distinct reproductive and sterile castes might proceed from the loss or acquisition of miRNA binding sites in the 3' UTRs of particular genes by drift or selection, coupled with divergent temporal or spatial expression of miRNAs between workers and queens. In fact, it has recently been suggested that miRNAs may be understood as contributing to canalization and genetic buffering of gene regulatory networks by interacting with transcription factors in coherent and incoherent feed-forward loops to stabilize phenotypic variability [28]. However, we need not posit that miRNAs act as direct switches for differential developmental pathways. The same canalizing effect could be achieved with miRNAs acting as global regulators of tissue identity and gene expression breadth and specificity. Indeed, the properties that make miRNAs attractive candidates as stabilizers of phenotypic variability would also allow miRNAs to modulate emergence of different phenotypes upon alternative spatial or temporal expression in different castes. Two candidates showed especially strong expression differences between identical tissues from bee queens and workers (Figure 1). Ame-mir-9a.F was expressed most strongly in worker versus queen thorax and abdomen. Candidate 5152a was overexpressed in queen versus worker head, then showed the opposite pattern in the abdomen.

We also present many unrecognized miRNAs in honey bee and show that some of them, as well as other canonical miRNAs, appear to be transcribed in a stage-, tissue- or caste-specific manner (Figure 1). In fact, the genomic location of many of the most strongly caste, stage or tissue biased miRNAs, coupled with known functional activities of some miRNAs in other species, orders and phyla, allow inferences regarding the roles these caste- or stage-biased miRNAs may play in honey bees. For instance, we find that ame-mir-9a is among the most strongly caste-biased miRNAs, with much higher expression levels in adult worker thorax and abdomen than similar queen tissues, but higher levels of mir-9a occur in queen pupae (Figure 1). Interestingly, mir-9a controls sensory organ precursors (SOPs) in Drosophila, with loss of mir9a function resulting in ectopic production of SOPs, while overexpression of mir-9a yields a severe diminution of SOPs. Mir-9a is also expressed at high levels in epithelial cells adjacent to SOPs in proneural clusters, suppressing sens through miRNA/target interactions in the sens 3' UTR, and inhibiting neuronal fate in non-SOP cells [29]. This suggests possible roles for ame-mir-9a in influencing caste differences in honey bees. Another example is $\mathrm{C} 1504 . \mathrm{F}$, which is expressed in higher levels in queens than workers (Figure 1) and is nested within the honey bee ortholog of the RNA binding protein gene, CG32062. Expression of CG32062 in Drosophila is dependent upon Notch-mediated signaling from the DorsoVentral organizer (D/V) boundary, and repressed by the homeotic gene, Ultrabithorax. The product of CG32062 likely constitutes a second long-range $\mathrm{D} / \mathrm{V}$ morphogen, independent of Wingless (Wg) [30]. MiRNAs in other organisms are often organized in clusters that lie in physical proximity in the genome, and may be present in multiple copies too. In D. melanogaster, the proapoptotic K-box miRNA mir-2, and mir-13 occur jointly. The same relationship holds in bees, and amemir-71 is also present within this same region (Table 1). In fact, even with a relatively fragmented genome consisting of over 9,00o scaffolds, we can discern that the honey bee harbors several linked sets and/or multiple copies of miRNAs. They include ame-mir-1, which is near ame-mir-133. We note that mir-1 and mir-133 are co-located in physical proximity in organisms as diverse as honey bees, frogs, mice and men, and are well-documented regulators of myogenesis in other organisms [31]. Ame-mir-1 and ame-mir-133 may exhibit similar functions in honey bees. Other examples of clustered miRNAs or multicopy miRNAs include: novel miRNA C $5152 \mathrm{a}$ antisense to $\mathrm{C}_{5152 \mathrm{~b}}$; novel $\mathrm{C}_{53} \mathrm{O} 3$ overlapping ame-mir-137; 
ame-mir-9b overlapping the ame-mir-79 locus, but on the opposite strand; ame-mir-12 near ame-mir-283; ame-mir275 near ame-mir-305; ame-mir-277 near ame-mir-317 and ame-mir-34; C1504 near ame-mir-375; and ame-let-7 on the same scaffold as ame-mir-100. Two of the most interesting cases involve multiple miRNAs in the introns of single genes. Ame-mir-277, ame-mir-317 and ame-mir-34 occur in the same intron of GB10191 - a core component of the RNA polymerase II complex. Finally, three copies of ame-mir-2, plus one instance each of ame-mir-13a and ame-mir-71, all occur within intron 3 of GB15727 - a serine/threonine phosphatase lost from Drosophila, but with both vertebrate and more ancient metazoan orthologs.

That fact that we found three GO terms ('Physiological process', 'Nucleus' and 'Response to stress') that were overrepresented among genes near miRNAs in both the Drosophila and bee genome demonstrates that some miRNAs function in the same or similar functions in Drosophila and bee. Furthermore, this result allows us to ascribe roles for honey bee miRNAs in processes relevant to these GO terms. Future studies of the specific genes near these miRNAs and annotated with these GO terms may help elucidate how these miRNAs function in honey bee.

The sensitivity of the GO experiment in bee was limited by a number of factors. The GO analysis considers only those bee genes with recognizable orthologs in Drosophila, and the GO annotation for bee genes was always based upon functional evidence from Drosophila. Furthermore, in honey bee, the GeneMerge E-score for GO terms present in every experiment varies somewhat depending upon the particular Drosophila ortholog selected for use in GeneMerge, at least when there is more than one Drsophila ortholog. While 'Development', 'Morphogenesis', 'RNA binding' and 'Signal transduction' were overrepresented in every Drosophila experiment at all distances, there are no GO terms overrepresented in every bee experiment at each distance. Therefore, we suggest that the lack of enrichment for these same GO slim terms in the bee experiment may reflect the lack of a complete gene list in honey bee, the paucity of direct functional evidence for honey bee genes, and the reliance upon Drosophila orthology and GO annotation for bee genes. As honey bee genome annotation and functional genomics proceeds, further GO analysis may reveal additional functional attributes for honey bee miRNAs.

\section{Conclusion}

Not surprisingly, the honey bee genome contains numerous candidate miRNAs that can be identified by computational methods. We show that some honey bee candidates identified in this way have been overlooked in other genomes. Some novel and canonical miRNA transcription levels differed strongly across the tested tissues and samples. Honey bees and other social insects are defined by a developmental poly- morphism between highly fertile, long-lived queens and largely sterile workers. Differences in miRNA expression observed in homologous tissues of queen and worker may help provide insights into gene regulation during the remarkable developmental switch characterizing caste differences in the honey bee.

\section{Materials and methods \\ Computational miRNA predictions}

Our first strategy for identifying novel miRNAs invoked BLASTN searches of known miRNAs from miRBase release 8.o [16] against the honey bee genome (Assembly release 4.0) using wordsize 7 and E-score threshold $\leq 0.1$. These searches identified several hundred candidate bee miRNAs with significant matches to miRNAs from other species. A sliding window of 110 nt with increments of 3 nt was scanned along the sequences extracted at $100 \mathrm{nt}$ upstream and downstream of each match. Windows were scored for folding energy (at least $25 \mathrm{Kcal} / \mathrm{mol}$ ) using RNAFOLD [32], then for base pairing and position of putative mature miRNA along the stem. Candidates with at least 16 bases paired to the opposite strand were considered putative mature regions. Windows that passed this scoring scheme were visually inspected for proper folding.

Our second strategy relied on three-way, all against all, genomic comparisons of D. melanogaster, A. gambiae and A. mellifera to identify probable honey bee miRNA candidates [17]. Hundreds of microconserved MCE sequences identified in this way included more than $40 \%$ of previously validated Drosophila miRNAs, and this set seems likely to contain additional and novel miRNAs shared by bee and Drosophila. The secondary structural features of known pre-miRNAs in Drosophila are expected to be characteristic of novel premiRNAs of bee as well, because the genes involved in processing primary RNA transcripts into mature miRNAs in Drosophila are conserved in honey bee. Consequently, secondary structures of candidate bee miRNA precursors were screened for proper folding and thermodynamic stability typical of Drosophila miRNA precursors, and putative mature miRNAs were eliminated if they did not lie within the stem regions of the pre-miRNA hairpins, according to the criteria previously proposed by Ambros et al. [33]. Ground-state energies and structures were computed with the Vienna Package [34].

For the third strategy we applied a novel algorithm, SLS, to the entire honey bee genome to identify sequences that would adopt appropriate hairpin secondary structure. In the SLS method, overlapping $100 \mathrm{nt}$ segments of the genome are analyzed for sequences that can form loops similar to those seen in known miRNAs. In detail, each 100 nt segment was aligned to its reverse complement using a modified Smith-Waterman alignment algorithm (G::T pairing was penalized less than other mismatches). Good alignments were tested to determine if they would form a stem and a loop with size typical of 
known miRNAs. Specifically, stems had to be $20-25 \mathrm{bp}$, and loops had to be 4-35 nt. Candidate sequences were then subjected to thermodynamic testing using Mfold [35] to determine free energy values. Those with folding energies less than $-20 \mathrm{kcal} / \mathrm{mole}$ were discarded. This entire process was performed on both the honey bee and Drosophila genomes. Putative miRNAs from honey bee that aligned well to putative miRNAs from Drosophila were saved as candidate miRNAs.

\section{Transcriptional analyses: $\mathbf{R T}-\mathbf{P C R}$}

RNA was extracted and enriched for short transcripts using a variant of the RNAqueous (Ambion, Austin, TX, USA) protocol. Honey bee tissues (head, thorax, and abdomen from queens and workers, and whole bodies from queen and worker prepupae) were ground in 200-600 $\mu \mathrm{l}$ lysis grinding buffer depending on tissue volume. This suspension was diluted in an equal volume of $64 \% \mathrm{EtOH}$ and then spun through the provided filter columns. The flow-through, containing smaller RNA species, was then mixed with a 70\% volume of isopropanol and passed through a second filter column in order to trap the now-precipitated small RNAs. After prescribed wash steps, RNA was eluted from this second column in $50 \mu \mathrm{l}$ sterile $\mathrm{H}_{2} \mathrm{O}$. RNA size range and quantity was estimated using an Agilent 9000 Bioanalyzer (Agilent Technologies, Santa Clara, CA, USA). A second extraction was carried out as above for queen and worker head, thorax, and abdomen, as well as third-instar larvae and prepupal bees. This extraction was separated using a 15\% denaturing (TBEurea) polyacrylamide gel (Invitrogen, Carlsbad, CA, USA). RNA species 18-30 nt in length were cut from the gel, eluted as a group using a FLASHPAGE mini-electrophoresis unit (Ambion), purified by EtOH precipitation, and resuspended in $50 \mu \mathrm{l}$ sterile $\mathrm{H}_{2} \mathrm{O}$.

Contaminating DNA was removed by exposing $2 \mu \mathrm{g}$ of each total RNA pool to $10 \mathrm{U}$ DNaseI with appropriate buffer (Ambion) in the presence of $20 \mathrm{U}$ RNAsin (Roche, Mannheim, Germany). Samples were incubated 1 hour at $37^{\circ} \mathrm{C}$, then $75^{\circ} \mathrm{C}$ for 15 minutes. Polyadenylated tails were added to all transcripts using a $15 \mu \mathrm{l}$ reaction containing $2 \mu \mathrm{g}$ total RNA, 2 U E-PAP enzyme with appropriate $1 \times$ buffer (Ambion), $4 \mathrm{mM} \mathrm{MnCl}_{2}$, and $1.7 \mathrm{mM}$ ATP. Samples were incubated at $37^{\circ} \mathrm{C}$ for 1 hour. cDNA was prepared from $0.4 \mu \mathrm{g}$ polyadenylated RNA template in a $15 \mu \mathrm{l}$ reaction containing 10 pmol oligo-dT linker (5'GCG AGC ACA GAA TTA ATA CGA CTC ACT ATA GGT ${ }_{12} \mathrm{VN}$ ) and $2 \mathrm{mM}$ dNTP. The reaction was heated to $70^{\circ} \mathrm{C}$ for 10 minutes and placed on ice. After preheating to $42^{\circ} \mathrm{C}$ for 2 minutes, $4 \mu \mathrm{l}$ of reverse transcriptase mix, containing 50 U Superscript II in appropriate buffer and reagents (Invitrogen) was added. Synthesis was carried out at $42^{\circ} \mathrm{C}$ for 50 minutes, followed by 15 minutes at $70^{\circ} \mathrm{C}$.

The above cDNA was diluted 1:5 and used as the template for amplification in an iCycler real-time PCR thermalcycler (Biorad, Hercules, CA, USA). Gene specific primers for approximately two thirds of the putative miRNAs were designed based on the predicted mature or precursor RNA sequences (Table 1). The $25 \mu \mathrm{l}$ reaction mixes consisted of $1 \mathrm{U}$ Taq DNA polymerase with appropriate buffer (Roche), $1 \mathrm{mM}$ dNTP mix, $2 \mathrm{mM} \mathrm{MgCl}_{2}, 1 \times$ SYBR Green dye (Molecular Probes, Eugene, Oregon, USA), $10 \mathrm{nM}$ Fluorescein calibration dye (Biorad), and $0.2 \mu \mathrm{M}$ of each forward and reverse primer. The thermal program for all reactions was $95^{\circ} \mathrm{C}$ for $30 \mathrm{~s}$ followed by 40 cycles of $\left(95^{\circ} \mathrm{C}\right.$ for $30 \mathrm{~s}, 60^{\circ} \mathrm{C}$ for $30 \mathrm{~s}, 72^{\circ} \mathrm{C}$ for $30 \mathrm{~s}$, $76^{\circ} \mathrm{C}$ for $10 \mathrm{~s}$ immediately after the extension step for fluorescence capture). Melt-curve analysis and agarose gel analyses were used to test whether PCR products were the appropriate size (gel products $60-80 \mathrm{bp}$, dissociation temperatures 76 $81^{\circ} \mathrm{C}$ ). In addition, qPCR runs using negative (no template) templates, as well as miRNA forward primers without the adaptor primer, were used to exclude primers that showed signs of spurious amplification $(n=23)$.

Threshold cycle $\left(\mathrm{C}_{\mathrm{T}}\right)$ values for each miRNA were subtracted from the mean $\mathrm{C}_{\mathrm{T}}$ values for all miRNAs surveyed in a given cDNA. Amplification efficiency (serial dilution) analyses suggested that these PCR reactions were highly efficient and, accordingly, relative abundances were calculated as $2^{\delta \mathrm{CT}}$. While the low replicate number precludes statistical analyses, means and standard deviations are presented for the two sample replicates in order to indicate sample variability.

\section{Gene ontologies of miRNA-regulated genes}

To determine the functional categories of bee and fly genes under control of miRNAs, we looked for GO terms [24] overrepresented among genes in close proximity to putative miRNAs in the Drosophila and bee genomes. GO slim terms and annotations for $D$. melanogaster genes generated at FlyBase [36] were obtained from the Gene Ontology Consortium website [37]. GO terms were assigned to genes of the honey bee Official Gene Set [18] using D. melanogaster orthologs, which were identified as described by the Honey Bee Genome Sequencing Consortium 2006 [15]. In cases where more than one fly ortholog existed for a given bee gene, a random fly ortholog was selected independently in each replicate experiment. GeneMerge [25] was then run using test sets of genes $<10 \mathrm{~kb},<20 \mathrm{~kb},<50 \mathrm{~kb}$ or $<100 \mathrm{~kb}$ from putative miRNAs and their associated GO slim terms, and a population set consisting of all mapped bee genes with fly orthologs (for the bee experiment) or all mapped fly genes (for the fly experiment). Ten replicate experiments were conducted for both fly and bee analyses, and only GO terms whose Bonferroni corrected E-scores were less than 0.05 in all ten replicate experiments were considered significantly overrepresented. For negative control experiments, GeneMerge was run on a test set of randomly selected bee or fly genes equal in number to the set of bee or fly genes $<10,<20,<50$ and $<100 \mathrm{~kb}$ from a putative miRNA. 


\section{Additional data files}

The following additional data are available with the online version of this article. Additional data file 1 provides a complete listing of candidate miRNAs, including miRBase designation, the sequence of the putative mature honey bee miRNA, the sequences $110 \mathrm{nt}$ up- and downstream of each mature miRNA candidate (putative precursor region), the genomic coordinates of each occurrence of mature and putative precursor miRNA sequences within the bee genome assembly release 4 , whether the candidate miRNA is intergenic, intronic, or overlapping a CDS, the GC content of the GC content domain in which the miRNA is embedded (described in [15]), and folding energy. Additional data file 2 provides alignments of primers to precursor miRNAs. Double strands of precursors are shown, with mature miRNA indicated as lower case embedded in the sense strand, which is otherwise uppercase. Note that reverse primers are shown in the 3 ' to 5 ' direction, to show alignment to the sense strand. Additional data file 3 provides qPCR expression results from gel-purified 18-30 nt RNA extractions from pooled tissues (head, thorax, and abdomen from queens and workers, and whole bodies from queen and worker prepupae). The ID column corresponds to gel lanes in part b in Additional data file 9. $\mathrm{C}_{\mathrm{T}}$ is a predetermined threshold at which fluorescence from PCR products exceeds background fluorescence. Additional data file 4 provides mean expression values for queen and worker honey bee tissue-specific samples. Additional data file 5 provides the primers employed in expression analyses, and expression estimates for pooled samples. Additional data file 6 provides miRNA tiling array probes with hybridization signal in the top $10 \%$ of tiling array results in two independent experiments. The RNA sample from array B had a higher concentration of bee brain and thorax than the first. Additional data file 7 provides GO analysis of genes located within 10, 20, 50 and $100 \mathrm{~Kb}$ of miRNAs. There is a different worksheet containing the results of 10 replicates each, for honey bee and Drosophila. Each worksheet provides E-score, Bonferroni Corrected E-score, and GO term. Additional data file 8 shows folded hairpins for precursors of novel honey bee miRNAs. Additional data file 9 provides two figures: Figure A shows a 15\% PAGE separation of small-enriched RNAs from honey bee queen head, thorax, and abdomen, and worker head, thorax, and abdomen. RNA sized at 18-30 nt was excised from the gel and purified for qPCR as described in the text. The left lane shows a 10 nt RNA size marker, with the $10 \mathrm{nt}$ band at bottom left. Figure B shows the size variation of PCR products generated from small-enriched RNA pools and candidate primers. Most products were approximately 75-90 bp in length. Alphanumeric label refers to sample ID as described in Additional data file 3. Additional data file 10 shows normalized expression across worker and queen samples for additional miRNA candidates. Values indicate relative expressions levels as $\log _{10}$ scale, with SD for three sample replicates, as described in the text.

\section{Acknowledgements}

We acknowledge funding from NIH 5-P4I-HG000739- I3, USDA ARS Special Cooperative Agreement 58-6204-6-039 and Bee Weaver Apiaries, Inc.

\section{References}

I. Lagos-Quintana M, Rauhut R, Lendeckel W, Tuschl T: Identification of novel genes coding for small expressed RNAs. Science 200I, 294:853-858.

2. Lau NC, Lim LP, Weinstein EG, Bartel DP: An abundant class of tiny RNAs with probable regulatory roles in Caenorhabditis elegans. Science 200I, 294:858-862.

3. Lee RC, Ambros V: An extensive class of small RNAs in Caenorhabditis elegans. Science 200I, 294:862-864.

4. Aboobaker A, Tomancak P, Patel N, Rubin GM, Lai EC: Drosophila microRNAs exhibit diverse spatial expression patterns during embryonic development. Proc Natl Acad Sci USA 2005, I02:18017-18022.

5. Giraldez AJ, Cinalli RM, Glasner ME, Enright AJ, Thomson JM, Baskerville $\mathrm{S}$, Hammond SM, Bartel DP, Schier AF: MicroRNAs regulate brain morphogenesis in zebrafish. Science 2005, 308:833-838.

6. Boehm M, Slack F: A developmental timing microRNA and its target regulate life span in C. elegans. Science 2005, 3 10:1954-1957.

7. Brennecke J, Stark A, Russell RB, Cohen SM: Principles of microRNA-target recognition. PLOS Biol 2005, 3:e85.

8. Krek A, Grun D, Poy MN, Wolf R, Rosenberg L, Epstein EJ, MacMenamin P, da Piedade I, Gunsalus KC, Stoffel M, Rajewsky N: Combinatorial microRNA target predictions. Nat Genet 2005 , 37:495-500.

9. Fahr KK, Grimson A, Jan C, Lewis BP, Johnston WK, Lim LP, Burge $C B$, Bartel DP: The widespread impact of mammalian microRNAs on mRNA repression and evolution. Science 2005, 310:1817-|82|.

10. Stark A, Brenneke J, Bushati N, Russel RB, Cohen SM: Animal microRNAs confer robustness to gene expression and have a significant impact on 3'UTR evolution. Cell 2005, 123: | |33-|| 46.

I I. Bartel DP: MicroRNAs: genomics, biogenesis, mechanism and function. Cell 2004, I | 6:28I-297.

12. Lim LP, Lau NC, Garrett-Engele P, Grimson A, Schelter JM: Microarray analysis shows that some microRNAs downregulate large numbers of target mRNAs. Nature 2005, 433:769-773.

13. Grun D, Wang YL, Langenberger D, Gunsalus KC, Rajewsky N: MicroRNA target predictions across seven Drosophila species and comparison to mammalian targets. PLoS Comput Biol 2005, I:el3.

14. Lai EC, Tam B, Rubin GM: Pervasive regulation of Drosophila notch target genes by GY-box-, Brd-box-, and K-box-class microRNAs. Genes Dev 2005, 19:1067-1080.

15. Honey Bee Genome Sequencing Consortium: Insights into social insects from the genome of the honeybee Apis mellifera. Nature 2006, 443:931-949.

16. Griffiths-Jones S, Grocock RJ, van Dongen S, Bateman A, Enright AJ: miRBase: microRNA sequences, targets and gene nomenclature. Nucleic Acids Res 2006, 34:D I40- I 44.

17. Tran T, Havlak P, Miller J: MicroRNA enrichment among short 'ultraconserved' sequences in insects. Nucleic Acids Res 2006 , 34:e65.

18. Elsik CG, Mackey AJ, Reese JT, Milshina NV, Weinstock GM: Creating a honey bee consensus gene set. Genome Biol 2007, 8:RI3.

19. Berezikov E, Cuppen E, Plasterk HA: Approaches to microRNA discovery. Nat Genet 2006, 38:S2-S7.

20. Shi R, Chang VL: Facile means for quantifying microRNA expression by real-time PCR. BioTechniques 2005, 39:5 19-525.

21. Stolc V, Samanta MP, Tongprasit W, Sethi H, Liang S, Nelson DC, Hegeman A, Nelson C, Rancour D, Bednarek S, et al.: Identification of transcribed sequences in Arabidopsis thaliana by using high-resolution genome tiling arrays. Proc Natl Acad Sci USA 2005, 1 02:4453-4458.

22. Sempere LF, Sokol NS, Dubrovsky EB, Berger EM, Ambros V: Temporal regulation of microRNA expression in Drosophila melanogaster mediated by hormonal signals and broad-Complex gene activity. Dev Biol 2003, 259:9-18.

23. Baskerville S, Bartel DP: Microarray profiling of microRNAs reveals frequent coexpression with neighboring miRNAs and 
host genes. RNA 2005, I I:24I-247.

24. Ashburner M, Ball CA, Blake JA, Botstein D, Butler H, Cherry JM, Davis AP, Dolinski K, Dwight SS, Eppig JT, et al.: Gene ontology: tool for the unification of biology. The Gene Ontology Consortium. Nat Genet 2000, 25:25-29.

25. Castillo-Davis $\mathrm{Cl}$, Hartl DL: GeneMerge - post-genomic analysis, data mining, and hypothesis testing. Bioinformatics 2003, 19:89|-892.

26. Evans JD, Wheeler DE: Expression profiles during honeybee caste determination. Genome Biol 2000, 2:research000 I.I-000 I.6.

27. Evans JD, Wheeler DE: Gene expression and the evolution of insect polyphenisms. Bioessays 200I, 23:62-68.

28. Hornstein E, Shomron N: Canalization of development by microRNAs. Nat Genet 2006, 38:S20-S24.

29. Li Y, Wang F, Lee J-A, Gao F-B: MicroRNA-9a ensures the precise specification of sensory organ precursors in Drosophila. Genes Dev 2006, 20:2793-2805.

30. Bajpai R, Sambrani N, Stadelmayer B, Shashidhara LS: Identification of a novel target of $D / V$ signaling in Drosophila wing disc: $\mathbf{W g}$ independent function of the organizer. Gene Expression Patterns 2004, 5: I|3-12I.

31. Rao PK, Kumar RM, Farkhondeh M, Baskerville S, Lodish HF: Myogenic factors that regulate expression of muscle-specific microRNAs. Proc Natl Acad Sci USA 2006, 103:8721-8726.

32. Denman RB: Using RNAFOLD to predict the activity of small catalytic RNAs. Biotechniques 1993, 15:1090-1095.

33. Ambros V, Bartel B, Bartel DP, Burge CB, Carrington JC, Chen $X$, Dreyfuss G, Eddy SR, Griffiths-Jones S, Marshall M, et al.: A uniform system for microRNA annotation. RNA 2003, 9:277-279.

34. Hofacker IL, Priwitzer B, Stadler PF: Prediction of locally stable RNA secondary structures for genome-wide surveys. Bioinformatics 2004, 20:186-190.

35. Zuker M: On finding all suboptimal foldings of an RNA molecule. Science 1989, 244:48-52.

36. FlyBase [http://flybase.org]

37. Gene Ontology Consortium [http://www.geneontology.org] 